\title{
Technological foresight: characterisation of research methods used in prospective analysis
}

Marcin ROMANOWSKI, Krzysztof NADOLNY

DOI: $10.30464 / j m e e .2018 .2 .2 .101$

Cite this article as:

Romanowski M., Nadolny K. Technological foresight: characterisation of research methods used in prospective analysis. Journal of Mechanical and Energy Engineering, Vol. 2(42), No. 2, 2018, pp. 101-108.

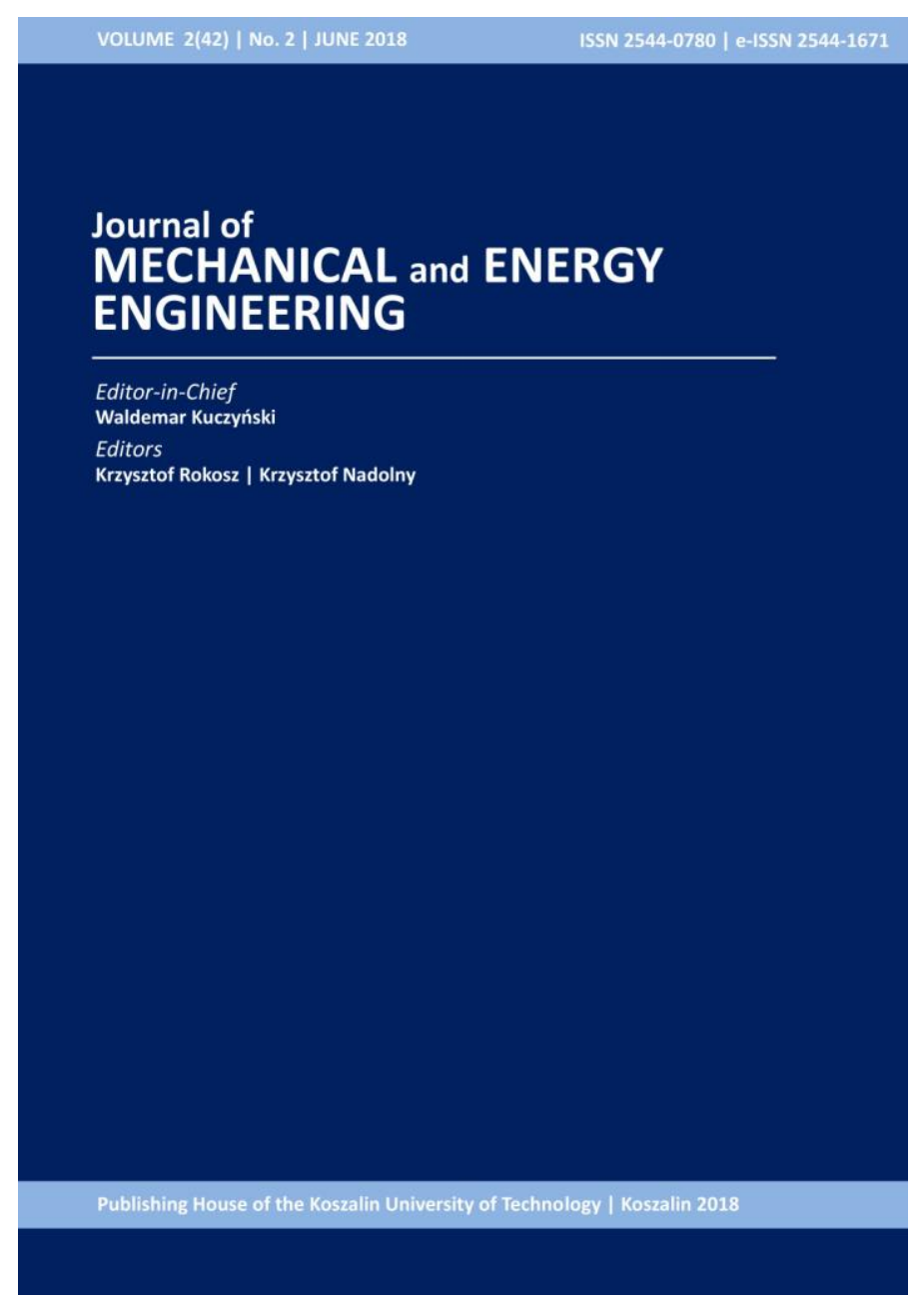

\section{Journal of Mechanical and Energy Engineering}

Website: jmee.tu.koszalin.pl

ISSN (Print): 2544-0780

ISSN (Online): 2544-1671

Volume: 2(42)

Number: 2

Year: 2018

Pages: 101-108

\section{Article Info:}

Received 28 February 2018

Accepted 14 April 2018

\section{Open Access}

This article is distributed under the terms of the Creative Commons Attribution 4.0 (CC BY 4.0) International License (http://creativecommons.org/licenses/by/4.0/), which permits unrestricted use, distribution, and reproduction in any medium, provided you give appropriate credit to the original author(s) and the source, provide a link to the Creative Commons license, and indicate if changes were made. 


\title{
TECHNOLOGICAL FORESIGHT: CHARACTERISATION OF RESEARCH METHODS USED IN PROSPECTIVE ANALYSIS
}

\author{
Marcin ROMANOWSKI ${ }^{1 *}$, Krzysztof NADOLNY ${ }^{2}$ \\ ${ }^{1 *}$ Faculty of Mechanical Engineering, Department of Production Engineering, Koszalin University \\ of Technology, Raclawicka 15-17, 75-620 Koszalin, Poland, e-mail: marcinro@wp.pl \\ ${ }^{2}$ Faculty of Mechanical Engineering, Department of Production Engineering, \\ Koszalin University of Technology, Poland
}

(Received 28 February 2018, Accepted 14 April 2018)

\begin{abstract}
The article presents the characteristics of a wide set of research methods used in prospective analysis known as foresight. Explorative and normative, quantitative and qualitative methods as well as techniques based on expert and assumptions were described in detail. The paper also describes the broader context of the notion of technological foresight and describes differences in this concept in relation to technology management.
\end{abstract}

\section{INTRODUCTION}

Forecasting manufacturing technology has become a major challenge in the global economy. Manufacturing companies place a very high emphasis on forecasting and saving, thus predicting which solution will be more advantageous. These challenges are twodimensional: on the one hand, they must be global in nature, which means new institutional arrangements for setting development priorities, and on the other hand they require a common search for alternative stabilisation systems. Foresight is an important process that provides a methodical basis for determining the future in production trends. The subject of the study is to present the essence of the methodology used in the foresight process and to show its importance in the activity of the enterprise [13]. In the Polish economy, the need to identify development tendencies in particular areas of science and economy, to create bold visions and development scenarios is particularly important. The task of quickly catching up with civilizational distance towards the most developed countries and regions can be accomplished mainly through innovative, anticipating and not imitation actions [17]. The culture of thinking about the future today is very diverse. Wasting waste, buying more than is needed or planning production stages wrongly are just some of the negative features of production management. This is unacceptable in large companies. These problems are common during production organisation. Overcoming such problems by management and anticipating what may be needed for a company in the near and distant future is essential for the proper functioning of the company. Technological foresight is an excellent research method. It combines the routine actions of managers with the prediction of technology that can be used in the future to make subsequent production steps faster and cheaper. This method is considered to be forwardlooking and very much needed in large companies [8].

\section{TECHNOLOGICAL FORESIGHT}

The term technology in Greek combines two components, $\tau \varepsilon \chi \eta v \varepsilon$ (techne) denoting art, craftsmanship, skill and $\lambda$ o science. However, in the simplest, basic approach, the science of manufacturing practice can be taken as technology [20].

Technological foresight is the most complex element of the technology development process. Luke Georghiou [3] describes technological foresight as "systematic means of evaluating those scientific developments and technologies" that can have a clear impact on industrial competitiveness, wealth creation and the quality of life. It allows data to be collected to formulate technology strategies that enable technological infrastructure development to be carried out. In addition, foresight of technology supports innovation and aid to enterprises in the field of technology management and leads to increased competitiveness. 
Technological foresight is gaining worldwide recognition as an excellent tool that helps to express views on future strategies of technology development. Its unique feature is the wide participation of a large number of stakeholders and experts, i. e. representatives of government, academia, industry and citizens. Technological foresight was established in Japan in 1970, but until the 1990s it was used in only a few countries. Since then, many other areas have undertaken technological foresight activities. Its primary goal is to identify future technologies, to create the future through actions taken today. Such actions may include: determining the order of investing in innovations or building new links between different markets [15]. It should be stated that the primary objective of technological foresight is primarily [9]:

- recognition of leading technologies;

- assessment of risks and opportunities for technology;

- analysis of the situation and technology development trends;

- discussion on the future of technology, taking into account the views of many circles;

- determination of key factors influencing the development of technology;

- determining the direction of technological development;

- consultation;

- identification of activities to create technology development.

There are four important aspects of the abovementioned definitions [8]:

- the attempt to look at the future must be systematic in order to be called foresight. This is a feature that distinguishes foresight from endogenous scripts that are created in everyday life;

- foresight refers to a longer period, which is usually seen as longer than the normal planning horizon. Therefore, foresight horizons are 5 to 30 years;

- technological progress should be balanced by the a market demand. Although this is a strict way of thinking about the process of innovation, technological foresight should not be dominated only by science and technology;

- foresight focuses on developing generic technologies, where there is a legal basis for government support. This is due to the lack of willingness of companies to finance strategic research.

The above aspects have so far been replaced by definitions of technological foresight, which put more emphasis on building the system and benefits of this process. Foresight comprises four key elements [15]:

- anticipating structures and projection of long-term technological progress and needs,
- interactive methods of research debates, analyses and research, including an extensive range of stakeholders, which is characteristic for foresight, as opposed to many traditional future studies, which are rather reserved for experts,

- interactive approaches include the creation of new social networks. The emphasis on the role of networking varies according to the technological foresight program,

- formal foresight products go beyond a presentation of scenarios and preparation of plans. The key element is the elaboration of a strategic vision, which is characterized by a sense of connection achieved through network processes.

Technological foresight is often identified with other forward-looking activities such as forecasting, future research or strategic planning. Foresight cannot be confused with more explicit predictions and makes assumptions about how the future will develop. Unlike foresight forecasts, foresight has no intention of predicting, but it is a process seeking common visions of the future, visions that the parties want to implement through actions taken at the moment. In this way, foresight is not connected with prediction of the future, but with its creation.

It should be noted that foresight does not replace forecasting, future research or strategic planning. Each of these activities has its own role. In many cases, the roles of these activities can interact.

A particular feature of this is the pressure exerted in a collective way in the framework of foresight. Such a perspective eliminates the identification of technological foresight as separate processes without attempting to build links between processes [9].

As noted by Halicka [6], foresight is a process which should be conducted as technological forecasting, systematically, in accordance with the adopted specific research procedure. The course of foresight research process depends on the research context. For example, O. Saritas [19] has identified five foresight phases: understanding, synthesis and modelling, analysis and selection, transformation and action.

The understanding phase consists in determining the scope of the survey, the premises and purposes and collecting input data for the whole process. The next phase is synthesis and modelling. It is a synthesis of the results of the scanning process in order to create models of the reality being studied. The third phase is responsible for analysis and selection, prioritisation and selection of alternative models for the future through negotiations with stakeholders. The next step is the transformation phase, which consists in defining the relationship between the future and the present. The last phase of the activities is formed by a framework for informing decision-makers about the necessity of structural and transformational changes [19]. 
Later, I. Miles [11] distinguished five stages of the foresight process consisting of the pre-design phase, the recruitment phase, the generation of knowledge, the activity and the renewal.

The process begins with the pre-project phase (pre-foresight), which consists in defining the research objectives, research methodology and establishing a research team. Then follows the recruitment phase, during which the project pages are identified, experts are recruited, research panels are created and organized. The third phase is to generate knowledge, which includes the acquisition and generation of knowledge, its analysis and synthesis, the codification of hidden knowledge, and the generation of new issues. In this phase, there is also a creation of the vision of the future. In the next stage, the process enters the action phase. It consists in converting the results obtained into real political, business or legal actions. The process ends the resumption phase. It includes monitoring and evaluation of the achievement of the objectives [11].

On the other hand, A. Magruk [9] developed 7 stages of technological foresight research illustrated in Figure 1 .

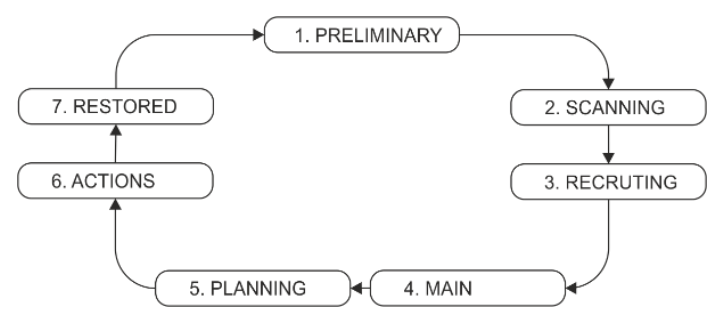

Fig. 1. PProcess of technological foresight process [9]

The process of technological foresight begins with the initial stage, which consists in justifying the research, determining the persons financing the research, its premises, scope and objectives, as well as the available resources, duration and time horizon of the research. The research methodology and study plan are also defined in this phase. The next stage is a scanning phase, which comes down to collecting information on the system, history and context of the research problem. The next stage is recruitment. It includes the identification of stakeholders, partners, steering team and experts. At this stage, a project team and research panels are created. Then the process enters the main stage, which consists in creating a new knowledge of the studied area and the vision of the desired future. The fifth phase is planning. It is based on the creation of strategic plans for the current decision-makers. Instead, the phase aims to transform reality into a desirable future. This phase builds on the existing and new networks between research performers and stakeholders and policy makers. Possible options, strategies and plans are being developed to take up the relevant directions of activity. The process ends with a re-launching phase consisting of learning, spreading results and building a foresight culture.

\section{TECHNOLOGICAL FORESIGHT VERSUS TECHNOLOGY MANAGEMENT}

Foresight research is one of the directions of development in management sciences and can take an ambiguous form. One of them is the technological foresight studies, which, according to Martin [10], are aimed at identifying emerging technologies or, according to Regera [16], at identifying and monitoring the so-called weak signals associated with new or existing technological solutions. This research direction was inspired by technological foresight, including predictions of the future characteristics of new technologies and their duration. However, in contrast to traditional foresight forecasting, however, it takes a more active form taking into account different variants of the future and consequences of particular actions, factors and decisions. Activities that constitute the basic technological context of foresight identify A. Magruk [9], which is presented schematically in Figure 2, while at the same time indicating that some of them refer to activities related to thinking about the future, while others are an analysis of the present state.

Starting from the general idea of technological foresight and technology management, one can see the convergence between these two issues. The common platforms identified for both issues are shown in Table 1.

Increasing technological knowledge intensifies the tendency to cooperate with the market for technology development, as the knowledge base of even the largest organisation is limited. This has an impact on the use of foresight approach in technology management, engaged cooperation or closer cooperation networks between science and business in order to increase the number of potential sources of innovations. It should be mentioned that this is one of the predictive approaches, complementing such issues as technological foresight, technology assessment and technology intelligence. For some time now there has been a tendency to compile these approaches under one term, the future-oriented technology analysis, which comprehensively captures both the diagnosis, as well as the prognosis of technology and its development dynamics, taking into account different time horizons. 


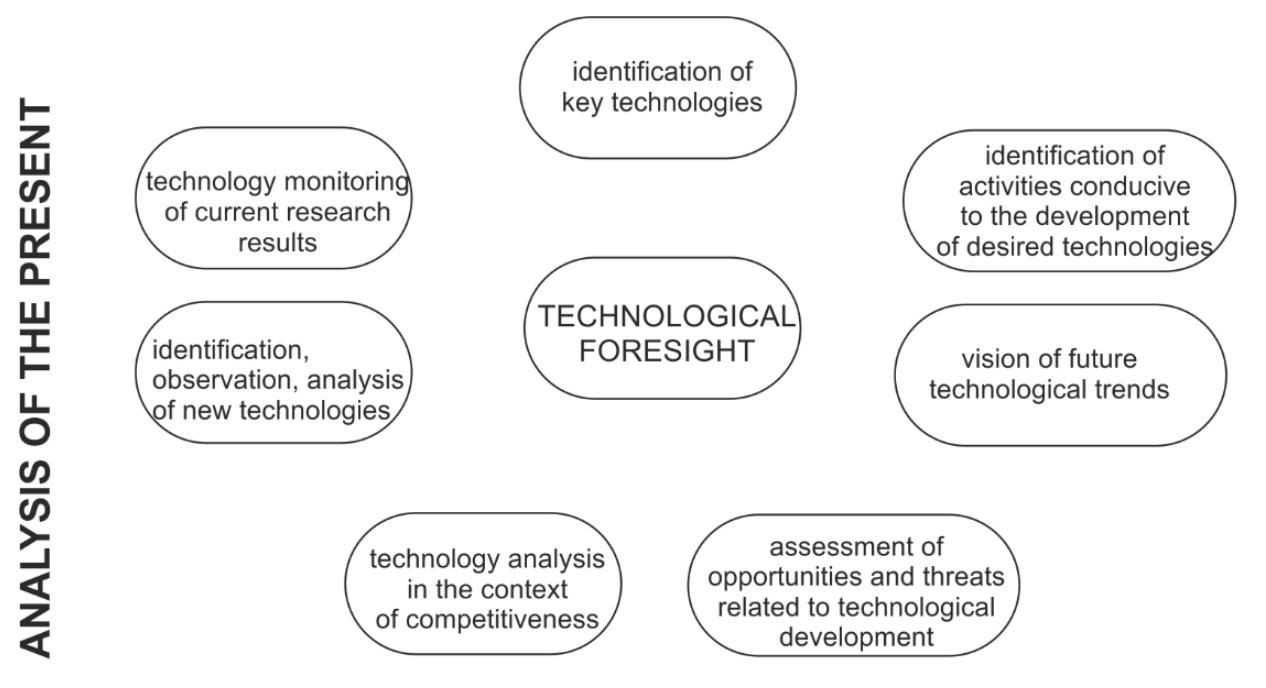

Fig. 2. Foresight research - technological context [5]

Tab. 1. Common Technology Foresight and Technology Management Platforms [5]

\begin{tabular}{|c|c|}
\hline TECHNOLOGICAL FORESIGHT & TECHNOLOGY MANAGEMENT \\
\hline $\begin{array}{l}\text { shaping the future in terms of technology and science; } \\
\text { analysing long-term trends; assessing the opportunities and } \\
\text { risks of technology development }\end{array}$ & $\begin{array}{c}\text { identifying opportunities and threats related to technology } \\
\text { development; making decisions on research and } \\
\text { development activities }\end{array}$ \\
\hline $\begin{array}{l}\text { identification of emerging technologies; analysis of } \\
\text { technological development measures }\end{array}$ & $\begin{array}{c}\text { understanding emerging new technologies; analysing } \\
\text { available resources, existing technologies, the future of the } \\
\text { market and the socio-economic environment }\end{array}$ \\
\hline $\begin{array}{l}\text { reinforcing areas of strategic research with high potential for } \\
\text { benefits; making more efficient choices; identifying key } \\
\text { technologies. }\end{array}$ & selecting technologies of strategic and tactical importance \\
\hline confront the lessons learned with public opinion & $\begin{array}{c}\text { consideration of stakeholders' needs and social interest } \\
\text { preference }\end{array}$ \\
\hline
\end{tabular}

\section{CHARACTERISATION OF FORESIGHT METHODS}

A special feature of foresight type research is the diversity of research methods used, ensuring different sources of origin and methods of data collection. This ultimately determines the quality of the input data. In addition to panel or program-based actions and inductive approaches, formal methods are likely to prevail. However, formal methods have some useful advantages [12]:

- striving to systematize the foresight process,

- increasing transparency of input materials, processes and starting materials,

- creation of hybrid forums for interaction and communication between different entities of the system,

- supporting the illustration of possible or desirable future events.

The other question is whether to use formal methods and how to use them. There are several possible limitations that are used to choose between formal methods. These include [12]:

- resources, especially time and money, are important factors in the choice of formal methods,

- large-scale surveys can be costly and time-consuming,

- desired width and depth of participation of experts and stakeholders in the foresight activity.

There are two key methods of foresight: explorative and normative [8].

Explorative methods begin in the present, which is a starting point and move towards the future or on the basis of extrapolation of past trends or random dynamics. This may also result from the "what if" question? on the implications of possible developments or events that may be outside known trends. Analysis of the impact trend and inter-effect analysis shows a conventional Delphi method. There are also some applications of the models included among the tools used [7].

The normative methods present an initial picture of possible and desirable future events or sets of future 
events of particular interest. There are therefore retroactive actions to demonstrate how future events could affect the present and when the available constraints, resources and technologies are known. The tools used here include various techniques developed in planning and related activities, such as cross-referencing trees and morphological analyses with the use of models and some less conventional, for example, the use of the Delphi method [2]. Recently, a method of using success scenarios and aspirational scenario workshops has been developed, where participants try to establish a common vision of the future, which is both desirable and credible [4];

The second important distinction is the distinction between quantitative and qualitative methods.

Quantitative methods are largely based on the numerical presentation of studies. They have many advantages, including the ability to study indicators and scale changes. They also have noticeable drawbacks, such as limited access to important social and political variables, the dangers of apparent precision, and communication problems with fewer target groups. Quantitative methods implicitly or explicitly use some kind of simple models. Models that are more complex together relate to variables, so their mutual influences can be tracked. Some quantitative approaches involve experts who give numerical values to events or create such values based on the number of people who agree with a particular statement or forecast such as the Delphi method [2].

Qualitative methods, on the other hand, are used where key trends or events are difficult to measure by means of simplified indicators or where quantitative data are not available. In addition, quality approaches encourage different forms of creative thinking such as brainstorming, utopian writing and science fiction. Methods for systematic work with qualitative data are becoming more and more available with the development of information technology, including tools for mind mapping and conversation analysis, which can also be helpful tools for facilitating meetings and workshops [8].

The third very important distinction is the distinction between methods that focus on examining and expressing experts' views and those that are based on the study of the consequences of assumptions. These are techniques based on expert opinions and assumptions [8].

Expert-based techniques shall seek to produce an information-based opinion and evidence which underpins the expert opinion. They seek to express views on the future and on trends and eventualities that may give rise to alternative events in the future and the objectives to be pursued. Such an approach may include either a large-scale survey of opinions (such as in the Delphi method) or a much smaller and more detailed development of visions (such as an analysis between influences, scenario workshops) [21].

Assumption-based techniques are those that show visions and priorities based on knowledge, which is usually generally available to the general public. Statistics and published analyses of probable breakthroughs and other events and eventualities are just some of the input data. Sometimes unavailability of related data may lead to special activities in order to generate a statistical link. These techniques benefit much more from expert knowledge than from interactive approaches. For example, technical competence is required to create a simulation model to describe the area of interest. Another example may be Delphi surveys, which are based on expert studies and produce quantitative results, but already some types of scenarios are mainly qualitative but largely based on assumptions [8].

The research methods used in foresight studies are often ordered using a foresight methodological diamond, taking into account four dimensions (representing four sources of knowledge) of foresight methods: creativity, interaction, facts and expertise, as shown in Figure 4 [14].

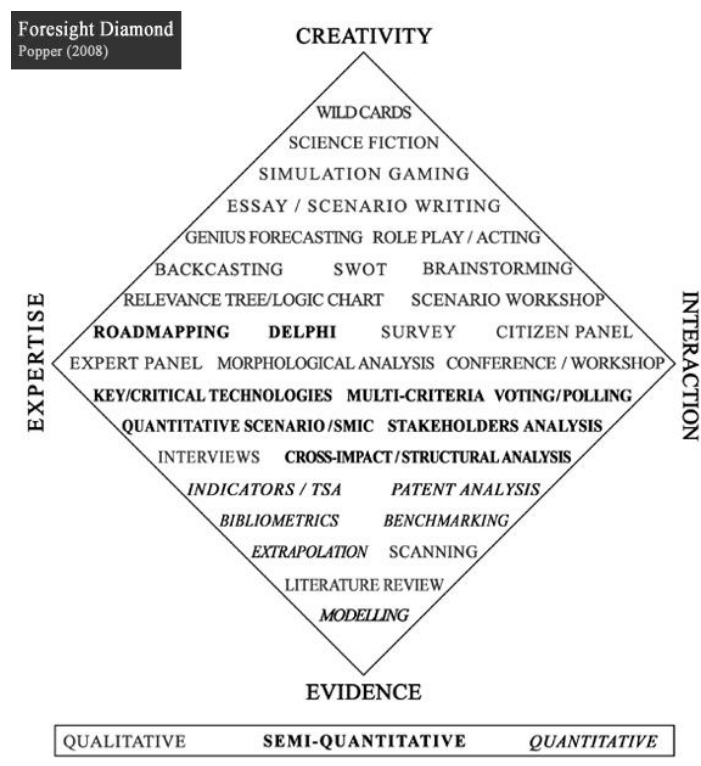

Fig. 3. Methodological diamond of foresight method - Test methods [14]

The most common research methods used in Poland include: scenario method, Delphi method, expert panels, SWOT analysis (B Strengths, Weaknesses, Opportunities, Threats) [7]. Moderately applied methods include brainstorming, cross-analysis of impacts, benchmarking, social consultations, literature review, technology development marshruths and surveys [14]. The least frequently used methods in Polish regional and sector foresight programs include interviews, modelling and simulations, stakeholder 
mapping, trend extrapolation, reference trees, sociometric studies, bibliometric analysis and problem hierarchical analysis [12]. Polish foresight programmes include, for example, the National Foresight Programme Poland 2020.

The literature review in foresight studies is one of the most important activities related to the analysis of the unknown state. A reliable literature review is a logical, reflexive approach based on the analysis of the current state of knowledge in the studied area. It is usually limited to a review of books, reports, journals, magazines, and websites in the subject matter covered by the research. According to Popper [14], "a good review of literature is usually characterised by a discursive style and transparent organisation of topics and related theories". The literature review for foresight research may also concern the review of future states of reality and visions of the future presented by various authors. Often literature review is linked to bibliometric analysis. bibliometric analysis, defined as quotation analysis, is understood as an examination of quantitative state and trends in literature development by statistical method on the basis of bibliographic descriptions or publishing statistics. It involves the use of a wide variety of scientific publication data to evaluate the results of national scientific activities, as well as to monitor scientific development, i. e. identify networks of research links, national and transnational, new, multidisciplinary fields of science and technology and to understand the internal logic of scientific development [12].

The method of expert panels, apart from literature review, is one of the most popular methods used in foresight research. It belongs to the group of heuristic methods, which are based on the opinions and intuition of experts. It is a particular type of method used in projects, aimed at building a vision of the development of a given area in the medium and long term perspective. Expert panels can be realised at local, regional, national or international level. They consist in organizing a group of people whose task is to analyse and synthesize the relevant knowledge for a given topic. Expert panels in practice boil down to regular meetings of a group of experts with high specialist knowledge, devoted to the sharing of knowledge on the subjects studied.

Brainstorming is a creative and interactive method whose idea manifests itself in the generation of new ideas, using casual associations, by creating creative potential in the members of the team and creating an atmosphere conducive to their generation. This method, used in expert panels, is a form of improving group decisions by encouraging free exchange of views and eliminating criticism. In the final part, valuable ideas are analysed in detail. The aim of brainstorming is to gather as many ideas as possible in a short space of time, which increases the chance of a valuable solution. There are three key phases in brainstorming: preparing the required conditions to ensure the best results, proposing different ideas for solving a given problem based on associations, as well as assessing suggestions, prioritising and grouping ideas. According to Popper [14], "brainstorming is a key component of foresight research, while its application is very flexible and can take the form of an unoriented discussion, as well as carefully prepared surveys".

Survey are one of the most popular methods used in social sciences, namely surveys. Survey research is the best available method for those researchers who want to collect original data to describe a population too large to observe directly. Owing to the appropriate random selection, a sample of respondents (sample) is obtained, which can be assumed to reflect the characteristics of the population and carefully constructed questionnaires provide data in the same form from all respondents. Surveys are a very good tool for measuring attitudes and views in a larger population [1].

The key technology approach consists in assessing the "criticality" (significance) of a specific technology (research direction/priority) on the basis of established criteria. The technologies examined to identify key technologies could, for example, be ranked in terms of attractiveness and feasibility (based on numerical valuation). In such a case, all the examined technologies are characterized by these two measurements, which allows for their graphical representation on a plane, facilitating the final interpretation. According to the UNIDO Technology Foresight Manual [8] technological foresight, the key/critical technologies method aims to provide a list of technologies recognized by appropriately selected experts as key technologies. The method involves four stages of research:

- location and selection of experts, in the form of narrow and/or broad consultations,

- the literature indicates that one can also refer to interviews with industry experts, environment scans or specialist research,

- shortening the initial list to the list of key technologies (for example, based on the ranking),

- preparation of the final list of key technologies.

It is important that the key technology approach can be used both for technology and for identifying key areas of research in different fields. In the context of foresight, however, there is no one way that could be described as just a right approach using the key technology method [8].

The method of technology mapping does not have an unambiguous, well-established definition, and the available literature allows only to distinguish the concept in the foresight methodology. In Polish foresight research, technology mapping is a term often 
associated with the creation of technology roadmapping, which is one of the most frequently used methods in foresight research, enabling the creation of multi-layered time charts, adapting technological solutions to market trends. However, as it results from the literature analyses carried out, these are separate but related methods. Technology development routes are an analysis of the future directions of technology development, and technology mapping is an in-depth diagnosis of its current state.

In the context of global foresight research, technology mapping is also not clearly defined, although it is more often identified in the adopted methodologies. Thus, technology mapping is defined as a characteristic of the research domain, i.e. an initial stage of the foresight process, having a significant impact on the structure of subsequent stages, a tool for developing strategic investments, or an instrument used in the first phase of foresight - the phase of understanding, including the creation of a relatively complete picture of the situation. It is also referred to as technology categorisation and classification used to review the analysis object and delineate its boundaries. The final effect of the method should be a graphical representation of selected technologies in the form of maps, allowing to indicate among other things the physical resources of technologies, their functionalities and relationships, without taking into account the time factor [18].

\section{CONCLUSIONS}

This article presents the term foresight, describes foresight methods and shows differences between technological foresight and technology management. These analyses allow the following general conclusions to be drawn.

1. Businesses are constantly looking for savings and new solutions to increase production efficiency. Newer and more competitive methods of market acquisition and profit making are being used. One of these methods is technological foresight.

2. The review of literature noted that foresight is often interchangeable with prospective technology analysis, which indicates a multi-criteria analysis of the issue.

3. The foresight process is responsible for the synergy of science and technology with business practice and identifies opportunities for the development of new technologies. It also allows the coordination of technological potential development with market scenarios.

\section{References}

1. Babbie E. (2007). Social research in practice, PWN, Warszawa. (in Polish)

2. Ciałkowska-Kuźmińska M. (2010). Delphi method. Sources, process, application in the assessment of psychosocial aspects of psychotherapy, Foundation for Mental Health Protection Foundation, Wrocław. (in Polish)

3. Georghiou L. (2003). The UK Technology Foresight Programme. Futures, vol. 28(4).

4. Gierszewska G., Romanowska M. (2002). Strategic analysis of the enterprise, Polish Economic Publishing House, Warszawa. (in Polish)

5. Gudanowska A. (2015). The essence of modern technologies in the context of technology management and technological foresight processes, Scientific research papers of the Silesian University of Technology. (in Polish)

6. Halicka A. (2016). Prospective analysis of technologies research methodologies and procedures, Publishing house of Bialystok University of Technology. (in Polish)

7. Kononiuk A., Magruk A. (2008). Overview of methods and research techniques used in foresight programmes, Science and Higher Education. (in Polish)

8. Krzewski A. (2007). United Nations Industrial Development Organization, technical and linguistic consultation., Unido Technology Foresight Manual, Polish Agency for Enterprise Development, Warszawa, 2007. (in Polish)

9. Magruk A. (2011). Innovative classification of technology foresight methods, Technological and Economic Development of Economy.

10. Martin B.R. (1995). Foresight in Science and Technology. Technology Analysis Strategic Management.

11. Miles I. (2002) Appraisal of Alternative Methods and Procedures for Producing Regional Foresight, Report prepared by CRIC for European Commission's DG Research founded STRATA - ETAN Expert Group Action, Manchester. (in Polish)

12. Nazarko J. (2011). Methodology and research procedures in the technological foresight project, Publishing house of Bialystok University of Technology, Bialystok. (in Polish)

13. Okon-Horodyńska E. (2006) Foresight: defining the future development of the national economy. Publishing House of the Polish Economic Society, Kraków. (in Polish)

14. Popper R., Keenan M., Miles I., Butter M., Fuenta G.S. (2007) Global Foresight Outlook 2007, The European Foresight Monitoring Network.

15. Porter Alan R. (2010) Technology Foresight: Types and methods, Georgia Institute of Technology.

16. Reger G. (2001). Technology foresight in companies: From an indicator to a network and process perspective. Technology Analysis Strategic Management.

17. Safin K. (2007-2013). Foresight as a method of shaping the future. Printer „Drukat”, Katowice. (in Polish)

18. Smith J. E. (2006) S\&T Foresight for Canadian Insight \& Strategic Preparedness, Office of the National Science Advisor, Canada.

19. Smith J. E., O. Saritas. (2011). Science and technology foresight baker's dozen: a pocket primer of comparative and combined foresight methods.

20. Tulley R.J. (2008) Is There Techne in My Logos? On the Origins and Evolution of the Ideographic Term Technology. "Int J Technol, Knowl Soc”, Vol. 4, No. 1, p. 93-104.

21. http://www.foresight.pl/metody-foresightu.html, (Access: February 2017) 


\section{Biographical notes}

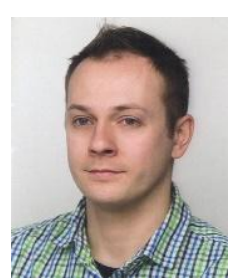

Marcin Romanowski received his M.Sc. degree in Exploitation Port and Fleet (specialisation: transport) from Maritime Academy in Gdynia. He works as Logistics and Procurement Manager in PRO-WAM Company in Koszalin and simultaneously studies at second year of Ph.D studies at the Faculty of Mechanical Engineering of Koszalin University of Technology. He is interested in cutting and milling technology as well as production cost minimalization and technological foresight.

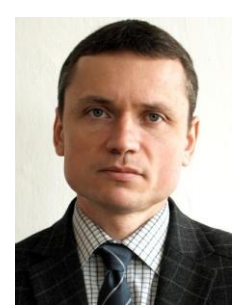

Krzysztof Nadolny received his M.Sc. degree in Mechanics and Machine Design and next Ph.D (with honors) as well as D.Sc. degree in Machinery Construction and Operation from Koszalin University of Technology, in 2001, 2006 and 2013, respectively. Since 2006 he has been a researcher in the Department of Production Engineering at the Koszalin University of Technology, where currently he works as an associated professor and head of research-didactic team for production planning and control. His scientific interests focus on problems concerning machining processes and tools, efficiency, monitoring and diagnostics of machining processes as well as tribology. He has participated in 2 international and 3 national research projects, presenting results of his work at 10 international and 21 national conferences, published more than 200 scientific papers in international and national journals, book chapters, as well as conference proceedings. He is also the author of 4 monographs and 9 national patents. 